Editorial

\title{
Highlights of WHO's work in the Eastern Mediterranean Region
}

Ala Alwan

This report provides highlights on the work of WHO in this Region in 2015 and the early part of 2016. It reflects the achievements made and the challenges encountered as well as the way forward and immediate next steps. It focuses in particular upon the five strategic priorities agreed by Member States in 2012: health systems strengthening towards universal health coverage; maternal and child health; noncommunicable diseases; health security and communicable diseases; and emergency preparedness and response.

Universal health coverage is the overarching goal of our work with Member States in strengthening health systems. It is a goal that was established by the WHO Regional Committee for the Eastern Mediterranean in 2012 and since then we have been supporting countries in implementing the actions included in the regional framework for action. The level of commitment to universal health coverage shown by Member States is high. In the past 18 months, WHO has supported countries in several areas that are key to moving forward on universal health coverage, including assessments in health financing, health policy and strategic planning, health sector regulation, and capacity development to support strengthening of public health laws and regulation. The regional framework for action, covering the four areas for universal health coverage (developing a vision and strategy; improving financial protection, expanding service coverage, and ensuring population coverage) will continue to be updated based on the needs of Member
States and will remain as a guide for technical support to countries and for monitoring progress.

The Regional Office also initiated strategic collaboration with an extensive network of international experts through the Disease Control Priorities 3 (DCP3) Project to develop a highpriority package of essential services for universal health coverage. The criteria used for inclusion of interventions in this package include evidence of impact, cost-effectiveness, and affordability. The progress of work in this initiative will be reviewed in a special session planned on the margins of the Regional Committee at its 63rd session.

Renewed focus was placed on developing workforce capacity, which is a major challenge across the Region. A series of consultations in the past 18 months have focused on developing a regional framework to implement the global strategy on human resources for health, endorsed by this year's session of the World Health Assembly, and a comprehensive strategic framework to strengthen nursing and midwifery which was also launched in the ministerial meeting in May 2016. A comprehensive review of medical education was undertaken, including an in-depth assessment of the situation in countries of the Region, conducted in collaboration with the International Federation of Medical Education, and active involvement of Member States. The Regional Committee subsequently endorsed a regional framework for action aimed at reform of medical education in the Region and this is planned to be discussed with ministers of health and higher education in a high-level meeting in 2017. To strengthen leadership in health and build public health capacity in Ministries of Health, a leadership course for senior health officials was established in 2015 , and successfully implemented in two rounds, in Geneva and Muscat in collaboration with the Harvard School of Public Health. More than 50 future health leaders have graduated and the course will continue to be conducted on annual basis. The Regional Office continues to host an annual seminar on health diplomacy for senior officials of ministries of health and foreign affairs, diplomats and parliamentarians.

Work continued in other areas of strategic importance to strengthening health systems, including regulation of the pharmaceutical sector and of medical devices, promotion of the family practice approach, regulating and partnering with the private sector, strengthening of hospital management and patient safety, civil registration and vital statistics, and health information systems. The Region now has the most comprehensive information available among allWHO regions of the situation with regard to civil registration and vital statistics systems. Based on the comprehensive assessments conducted in most Member States, a regional strategy to strengthen civil registration including reporting of cause specific mortality is now available to guide countries in implementing priority actions.

WHO continued to support implementation of the regional framework on health information systems and use of 
the core indicators and to build capacity to strengthen collection, reporting and dissemination of data. A practical tool has been developed to assess the gaps in the capacity of countries in generating and using reliable data for the 68 regional core health indicators. The assessment will be used to recommend actions to address the gaps and strengthen health information systems. Comprehensive health profiles, which document the current situation, challenges, gaps, opportunities and way forward in each health programme, have also been developed and updated in collaboration with Member States. Every country now has a brief sheet containing the key health indicators as well as an outline of the strengths, potential weaknesses, challenges and priorities for its own health system. These profiles are updated annually in close consultation with the Ministry of Health.

The road map for universal health coverage endorsed by the Regional Committee in 2014 will continue to guide our work in strengthening health systems. We will continue to focus on building capacity in leadership and governance. Health policy analysis and planning, health legislation and regulation, and continued strengthening of health information systems, including civil registration and vital statistics, will remain important areas of work. Strategic guidance on health workforce development will be finalized. Priority will be given to supporting countries in implementing the strategic framework on nursing and midwifery while the planned high-level meeting of ministers of health and medical education is expected to be particularly important in shaping the future of regional reform in medical education.

By the end of the year it was clear that the Region had made significant reductions in maternal and child mortality since 1990, as shown by the latest monitoring data for the Millennium Development Goals. However, the levels of reduction fell short of meeting the overall regional targets of MDGs 4 and 5. The regional initiative on saving the lives of mothers and children continued to target the main challenges in countries with a high burden of maternal and child deaths. The nine high-burden countries have launched maternal and child health acceleration plans, initially aimed at improving outcomes towards the MDG targets, but also preparing the way for the Sustainable Development Goals (SDGs) set for 2030. WHO also focused on quality of care, including preconception and neonatal care, which are becoming increasingly important elements of maternal and child survival in the Region.

Implementation of the United $\mathrm{Na}$ tions global strategy and the relevant SDG3 targets will be important drivers of progress in women's, children's and adolescents' health. We will continue to focus on building capacity in countries to end preventable deaths among women, children and adolescents. In this regard, WHO will focus on supporting and monitoring progress in, and the implementation of action plans on, reproductive, maternal, newborn and child health. Special emphasis will be placed on strengthening preconception and neonatal care. A list of evidencebased public health interventions for preconception care, including highimpact interventions for the prevention and control of congenital and genetic disorders, will be finalized for recommendation to countries by the end of 2016.

Nutrition remains an area of concern. A regional roadmap was developed for countries to implement the global targets set by the World Health Assembly in 2012 and the recommendations of the Second International Conference on Nutrition (ICN-2). Most countries now have national strategies or action plans. The regional policy statement on the urgent need to fully implement the International Code of Marketing of Breast Milk Substitutes was promoted while the number of nutrition stabilization centres for treatment of severe and complex cases of malnutrition was expanded in countries in emergency situations.

Noncommunicable diseases remain the biggest cause of premature death in the Region, in particular heart disease, cancer, chronic lung disease and diabetes. Despite high-level political commitments to action and some impressive achievements by some countries, progress has generally been inadequate, patchy and uneven. Tobacco use is still increasing, the rates of overweight and obesity in both adults and children are alarmingly high in most countries and there is no evidence that trends of other risk factors, including physical inactivity, are improving.

We continued to focus on supporting countries to implement the Political Declaration of the 2011 High-Level Meeting of the United Nations General Assembly on the Prevention and Control of Non-Communicable Diseases, through the regional framework for action endorsed by the Regional Committee in 2012. At global level, the Regional Office played an important role in advocacy for an accountability framework to measure progress, while the contribution of countries was substantial in ensuring that the 10 indicators crafted globally to measure the progress made by Member States were aligned with the indicators of the regional framework for action. Countries are now better able to monitor and report on progress in relation to the time-bound commitments set by the Political Declaration.

The regional framework addresses prevention and control through four areas: governance, surveillance, prevention and health care. In 2015, we provided each Member State with the first issue of an annual brief profile of its national response, based on the 10 process indicators. This enabled countries to see at a glance the progress made in implementation. So far, less than a third of countries have national multisectoral strategies or plans for the prevention 
and control of noncommunicable diseases, or have set national voluntary targets, which are essential first steps. We conducted extensive advocacy in 2015 and early 2016 to highlight the importance of these issues.

A number of key actions were also taken at regional level to support countries in a multisectoral response. These included the development, in collaboration with the WHO Collaborating Centre at Georgetown University, of a dashboard and policy briefs on best practices in health legislation for noncommunicable diseases, as well the publication of policy guidance on reducing dietary intake of sugar, complementing the policy guidance on reducing dietary intake of salt and fat. Support was also provided to countries to update tobacco control legislation and to build capacity for promotion of physical activity.

Full engagement of non-health sectors will be crucial in ensuring continued progress in implementing the regional framework for action to implement the United Nations Political Declaration on noncommunicable diseases. WHO will continue to work with Member States to achieve the progress needed by the time of the next United Nations review in 2018, but such progress will basically depend on the commitment and actions of Member States.

Also in 2015, the Regional Committee endorsed a practical and evidencebased regional framework to scale up action on mental health and operationalize the comprehensive action plan for mental health 2013-2020. Four domains of action were identified by the regional framework: governance, prevention, health care and surveillance.

Important progress was made in 2015 to improve health security in the Region. Following the rapid external assessments conducted by the Regional Office at the end of 2014 and beginning of 2015, of Member States' capacity to detect and respond to a case of Ebola virus disease, it became clear that countries' readiness to fully implement the capacities required under the International Health Regulations (IHR 2015) was considerably lower than was reported through self-assessment. In 2015, the Regional Committee decided therefore to establish a regional assessment commission to facilitate and provide technical guidance to countries and to oversee a process of independent joint external evaluation, a shift from self-assessment, in order to objectively assess the country's capacity to prevent, detect and respond to health security threats. At the same time it called for harmonization of the existing assessment tools available, as a result of which our region became a leading player in the development of the joint external evaluation (JEE) tools which are now accepted globally by all parties concerned.

Joint external evaluation for IHR capacities, using the new tools, has been conducted in four countries in the Region so far and a plan has been developed to conduct such independent and objective assessments in at least 10 countries by the end of 2016 and all countries by the end of 2017. This is a very important step forward. Nevertheless, serious challenges exist to the efforts to prevent and control emerging and re-emerging diseases and all countries will have to reinforce their capacities in dealing with threats to health security. Over the next 18 months the work will continue in order to ensure that all Member States are evaluated, that action plans are prepared based on the evaluation outcomes and that implementation to address the gaps is begun without delay.

Outbreaks of communicable disease were a continuing threat throughout 2015 as conflict and population displacement continued to escalate, posing challenges for maintenance of immunization coverage as well as other essential and life-saving public health services. Our response has been to continue to focus, and double our efforts, on helping Member States to establish effective and timely disease surveillance, for example- expanding the early warning, alert and response network (EWARN) in crisis-affected countries and enhancing the early warning surveillance system for influenza, respiratory diseases and other infectious diseases, in order to early detect and rapidly respond to these health threats. Lack of access to insecure areas to implement appropriate control interventions resulted in outbreaks of dengue fever in Sudan and Yemen, and cholera in several different countries. Nevertheless, the rapid detection and containment of these outbreaks, particularly a cholera outbreak in Iraq, as well of the frequent hospital outbreaks of MERS in Saudi Arabia, are some of the impressive examples of WHO's timely and effective response, thus preventing any major international health emergency from the continuing and widening threats to health security in the Region.

Good progress was made with regard to eradication of polio in the two remaining endemic countries of Afghanistan and Pakistan, although there are still areas where poliovirus continues to circulate. By mid May 2016, all countries had made the globally required switch from trivalent to bivalent oral poliovirus vaccine for routine and campaign use. The positive progress in polio eradication gives great cause for hope. There is now a great deal of optimism that the work we are doing in this region is now at a turning point for finishing the job of global polio eradication. We must continue to maintain our support for the programmes in Afghanistan and Pakistan and the highest commitment to ensuring all children are immunized everywhere. Likewise, in order to prevent outbreaks we must, and will, continue to ensure provision and maintenance of immunization coverage for all childhood diseases in emergency contexts, where many children remain hard to reach. 
The Regional Committee endorsed the Eastern Mediterranean vaccine action plan, as a framework for implementation of the global vaccine action plan, as well as the regional malaria action plan 2016-2020 for implementation of the global technical strategy for malaria 2016-2030. We convened and engaged a broad range of stakeholders in the development of a regional action plan for viral hepatitis.

2015 saw a significant deterioration in the humanitarian situation in the Eastern Mediterranean Region. The crisis in Yemen was designated a highest threat level (Level 3) emergency by the United Nations in July 2015 and the Region now hosts three Level 3 emergencies, including the crises in Iraq and the Syrian Arab Republic. Overall throughout the Region, more than 62 million people were in need of health services as a result of emergencies by the end of 2015, placing a significant strain on already weakened and overwhelmed health systems. More than $60 \%$ of all refugees and internally displaced persons originate from the Region; in Lebanon, refugees now comprise a third of the total population.

Shortages in specialized medical staff, medicines and medical equipment, and other health resources, especially in areas where access for $\mathrm{WHO}$ and partners was limited, had severe impact on the delivery of health services, contributing to the deterioration in the health status of some populations and an increased number of preventable deaths. Despite resource limitations and the major gaps in humanitarian funding, our regional emergency response programme was restructured in 2015 and considerably strengthened to deal with the unprecedented increase in the number, magnitude and severity of crises requiring WHO's support. Our capacity in leading humanitarian health relief has been reinforced and we continued to prioritize the $\mathrm{WHO}$ response to crisis in the Region ensuring the provision of essential medicines, supplies and humanitarian aid, as well strengthening disease surveillance, immunization campaigns, trauma care services, management of chronic diseases and mental health support. We continued to seek and implement approaches and strengthen partnerships to ensure people trapped in hard-to-reach areas were able to receive health care wherever possible, and to support mobile health services.

It is clear, and has been highlighted by the Regional Committee in a number of sessions, that we need to ensure a more systematic and effective approach, both within countries and across the Region, to responding to the health needs of populations affected by conflict and emergencies and to ensuring that health systems in all affected countries are strengthened and continue to deliver urgently needed health services to displaced populations and host communities. This will require improved and expanded coordination, action and engagement from other sectors and stakeholders including the affected communities themselves.

Following the endorsement in May 2016 by the World Health Assembly of the new WHO health emergencies programme, additional restructuring has taken place and a substantial increase in human resources is already planned.

The Regional Emergency Solidarity Fund was activated in January 2016 to ensure predictable financing of surge/rapid response to natural and man-made disasters in the Region and the WHO logistics hub, established in Dubai's International Humanitarian City to ensure the timely provision of critically needed medicines, medical supplies and medical equipment to countries in the Region and beyond, was operationalized. I hope that Member States in the Region will continue to support resource mobilization efforts for humanitarian and health support in affected countries, as well as in providing much needed technical and response capacity.
Throughout the past 18 months we have continued to complement our technical and operational activities with parallel actions to strengthen managerial effectiveness. This included continued emphasis on accountability, transparency and efficiency, and on strengthening the WHO workforce, especially at country level. Compliance is now monitored through a monthly compliance dashboard and is tied in with performance appraisal mechanisms. We increased focus on capacity-building initiatives, such as an integrated training programme for budget centres, dedicated compliance forums and outreach initiatives. This has yielded positive initial results in terms of improved management, compliance and accountability, including a decrease of over $80 \%$ in overdue reports on direct financial contributions compared with 2013, a decrease of over $80 \%$ in overdue donor reports compared with 2014 and closure of the majority of outstanding external and internal audit recommendations. A project was also completed to address non-compliance in non-staff contractual modalities.

In keeping with the practice of the past few years, a high-level meeting for ministers and representatives of Member States and permanent missions in Geneva was held prior to the World Health Assembly. These meetings continue to provide an opportunity to review, with ministers of health and senior government officials, progress in addressing key priorities since the previous Regional Committee and to strengthen Member States' engagement in global discussions on health and WHO reform.

There is clear evidence that this region is leading in several programme areas within WHO. The work we are doing in health system strengthening, health security and noncommunicable diseases has laid the groundwork for many years to come and provides a strong basis for countries to move forward with confidence. It is also 
increasingly recognized in our region that collaboration between sectors is essential for the achievement of long-term health and development goals. Nevertheless, there is still a long way to go. As we move forward into the post-2015 era and Member States initiate work towards the SDGs, there are four key areas that will receive attention in our work: advocacy for the SDG health targets; harmonization of existing WHO health strategies with the SDG targets; identification of more effective mechanisms for multisectoral action both within countries and at the regional and global levels; and strengthening of health information systems to support progress monitoring. 2016-2017 will be crucial in laying this foundation. 